\title{
Total least squares methods
}

\author{
Ivan Markovsky, ${ }^{1}$ Diana M. Sima, ${ }^{2}$ and Sabine Van Huffel ${ }^{2 *}$
}

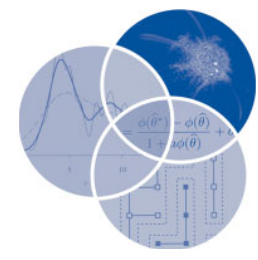

Recent advances in total least squares approaches for solving various errorsin-variables modeling problems are reviewed, with emphasis on the following generalizations:

1. the use of weighted norms as a measure of the data perturbation size, capturing prior knowledge about uncertainty in the data;

2. the addition of constraints on the perturbation to preserve the structure of the data matrix, motivated by structured data matrices occurring in signal and image processing, systems and control, and computer algebra;

3. the use of regularization in the problem formulation, aiming at stabilizing the solution by decreasing the effect because of intrinsic ill-conditioning of certain problems.

(C) 2009 John Wiley \& Sons, Inc. WIREs Comp Stat 20102 212-217

$\mathrm{F}$

or extensive reviews of the total least squares (TLS)

approach and its applications, we refer the reader to the following

- Overview papers: Refs 1-4;

- Proceedings and special issues: Refs 5-8; and

- Books: Refs 9-10.

The focus of this paper is on computational algorithms for solving the generalized TLS problems. The reader is referred to the errors-in-variables literature for the statistical properties of the corresponding estimators, as well as for a wider range of applications.

\section{WEIGHTED AND STRUCTURED TOTAL LEAST SQUARES PROBLEMS}

The TLS solution

$$
\begin{aligned}
\widehat{x}_{\mathrm{tls}}= & \underset{x, \widehat{A}, \widehat{b}}{\arg \min _{1}}\left\|\left[\begin{array}{ll}
A & b
\end{array}\right]-\left[\begin{array}{ll}
\widehat{A} & \widehat{b}
\end{array}\right]\right\|_{\mathrm{F}} \\
& \text { subject to } \widehat{A} x=\widehat{b}
\end{aligned}
$$

\footnotetext{
*Correspondence to: sabine.vanhuffel@esat.kuleuven.be

${ }^{1}$ School of Electronics and Computer Science, University of Southampton, Southampton, UK

${ }^{2}$ Department of Electrical Engineering, Katholieke Universiteit Leuven, Belgium
}

DOI: $10.1002 /$ wics.65 of an overdetermined system of equations $A x \approx b$ is appropriate when all elements of the data matrix $\left[\begin{array}{ll}A & b\end{array}\right]$ are noisy and the noise is zero mean, independent, and identically distributed. More precisely, (under regularity conditions) $\hat{x}_{\text {tls }}$ is a consistent estimator for the true parameter value $\bar{x}$ in the errors-in-variables (EIV) model

$$
A=\bar{A}+\widetilde{A}, \quad b=\bar{b}+\widetilde{b}, \quad \bar{A} \bar{x}=\bar{b},
$$

where the vector of perturbations $\operatorname{vec}\left(\left[\begin{array}{ll}\tilde{A} & \tilde{b}\end{array}\right]\right)$ is zero mean and has covariance matrix that is equal to the identity up to a scaling factor, i.e.,

$$
\begin{gathered}
\mathrm{E}\left(\operatorname{vec}\left(\left[\begin{array}{ll}
\widetilde{A} & \tilde{b}
\end{array}\right]\right)\right)=0 \quad \text { and } \\
\operatorname{cov}\left(\operatorname{vec}\left(\left[\begin{array}{ll}
\widetilde{A} & \tilde{b}
\end{array}\right]\right)\right)=\sigma^{2} I .
\end{gathered}
$$

The noise assumption (3) implies that all elements of the data matrix are measured with equal precision, an assumption that may not be satisfied in practice.

A natural generalization of the EIV model (Eq. $(2,3))$ is to allow the covariance matrix of the vectorized noise to be of the form $\sigma^{2} V$, where $V$ is a given positive definite matrix. The corresponding estimation problem is the TLS problem (1) with the Frobenius norm $\|\cdot\|_{\mathrm{F}}$ replaced by the weighted matrix norm

$$
\|\Delta D\|_{V^{-1}}:=\sqrt{\operatorname{vec}^{\top}(\Delta D) V^{-1} \operatorname{vec}(\Delta D)}
$$


i.e.,

$$
\min _{x, \widehat{A}, \widehat{b}}\left\|\left[\begin{array}{ll}
A & b
\end{array}\right]-\left[\begin{array}{ll}
\widehat{A} & \widehat{b}
\end{array}\right]\right\|_{V^{-1}} \quad \text { subject to } \quad \widehat{A} x=\widehat{b} .
$$

In [Ref 11, Section 4.3] this problem is called weighted total least squares (WTLS). Closely related to the WTLS problem are the weighted lowrank approximation problem ${ }^{12,13}$ and the maximum likelihood principal component analysis problem. ${ }^{14,15}$

As opposed to the weighted least squares problem, which is a trivial generalization of classical least squares, the WTLS problem does not have, in general, a closed form solution similar to the one of the TLS problem. The most general WTLS problem with analytic solution has a weight matrix of the form $V^{-1}=V_{2}^{-1} \otimes V_{1}^{-1}$, where $\otimes$ is the Kronecker product, $V_{1}$ is $m \times m$, and $V_{2}$ is $(n+1) \times(n+1)$ ( $m$ is the number of equations and $n$ is the number of unknowns in $A x \approx b$ ). For general weight matrix, the problem can be solved by local optimization methods. However, there is no guarantee that a globally optimal solution will be found.

There are two main categories of local optimization methods for solving WTLS problems: alternating projections and variable projections. ${ }^{16}$ They are based on the observation that the constraint of the WTLS problem (4) is bilinear, which implies that the problem is linear in either $x$ or $\widehat{A}$ and, therefore, can be solved globally and efficiently. Alternating projections is an iterative optimization algorithm that on each iteration step

1. solves a (linear) least squares problem in an $n \times(n+1)$ extended parameter $X_{\text {ext }}$ with $\widehat{A}$ fixed to the value obtained on the previous iteration step:

$$
\min _{X_{\mathrm{ext}}}\left\|\left[\begin{array}{ll}
A & b
\end{array}\right]-\widehat{A} X_{\mathrm{ext}}\right\|_{V^{-1}},
$$

2. solves a least squares problem in $\widehat{A}$ with $X_{\text {ext }}$ fixed to the optimal value of Eq. (5)

$$
\min _{\widehat{A}}\left\|\left[\begin{array}{ll}
A & b
\end{array}\right]-\widehat{A} X_{\mathrm{ext}}\right\|_{V^{-1}} .
$$

The parameter $x$ is recovered from $X_{\text {ext }}$, as follows

$$
\begin{aligned}
& x:=X_{\mathrm{ext}, 1}^{-1} x_{\mathrm{ext}, 2}, \\
& \text { where } X_{\mathrm{ext}}=\left[\begin{array}{ll}
\stackrel{n}{X_{\mathrm{ext}, 1}} & \stackrel{1}{x_{\mathrm{ext}, 2}}
\end{array}\right] .
\end{aligned}
$$

In the statistical literature, the alternating projections algorithm is given the interpretation of expectation maximization (EM). The problem of computing the optimal approximation $\widehat{A}$ given $X_{\text {ext }}$ is the expectation step and the problem of computing $X_{\text {ext }}$, given $\widehat{A}$ is the maximization step of the EM procedure.

The variable projections method uses the closedform solution of the expectation problem (6):

$$
\begin{aligned}
& f(x) \\
& :=\sqrt{d^{\top}\left(V^{-1}-V^{-1} \mathbf{X}_{\mathrm{ext}}^{\top}\left(\mathbf{X}_{\mathrm{ext}} V^{-1} \mathbf{X}_{\mathrm{ext}}^{\top}\right)^{-1} \mathbf{X}_{\mathrm{ext}} V^{-1}\right) d,},
\end{aligned}
$$

where

$$
d:=\operatorname{vec}\left(\left[\begin{array}{ll}
A & b
\end{array}\right]\right) \quad \text { and } \quad \mathbf{X}_{\mathrm{ext}}:=\left[\begin{array}{ll}
I_{n} & x
\end{array}\right] \otimes I_{m} .
$$

This is a projection of the rows of $\left[\begin{array}{ll}A & b\end{array}\right]$ on the subspace perpendicular to $\left[\begin{array}{c}x \\ -1\end{array}\right]$. The minimization over $x$ is then an unconstrained nonlinear least squares problem $\min _{x} f(x)$, which can be solved by standard optimization methods, e.g., the Levenberg-Marquardt method.

Another generalization of the TLS problem (1) is to add constraints on the approximation matrix $\left[\begin{array}{ll}\widehat{A} & \widehat{b}\end{array}\right] \cdot{ }^{17}$ Such constraints are needed in applications where the data matrix is structured and the approximation is required to have the same structure. For example, in signal processing the output $y$ of a finite impulse response (FIR) system to an input $u$ is given by multiplication of a Toeplitz matrix constructed from $u$ by the vector of the impulse response samples. In an FIR system estimation problem, where both the input and the output are noisy, the approximation matrix is required to have Toeplitz structure for the result to have interpretation as a description of an FIR system.

Similar to the WTLS problems, in general, structured total least squares (STLS) problems ${ }^{11}$ have no analytic solution in terms of the singular value decomposition (SVD) of the data matrix. Beck and Ben-Tal ${ }^{18}$ solved globally STLS problems with block-circulant structure by using the discrete Fourier transform and the solution of standard TLS problems. For other types of structure one has to resort to local optimization methods. In case of linearly structured problems, the constraint of the STLS optimization problem is bilinear, so that the alternating projections and variable projections methods, similar to the ones developed for the WTLS problem, can be used. 


\section{REGULARIZED AND TRUNCATED TOTAL LEAST SQUARES PROBLEMS}

Linear approximation problems $A x \approx b$ are considered ill-posed when small variations in the data $A$ and $b$ lead to large variations in the computed solution $x$. In the context of ordinary least squares, methods such as ridge regression, Tikhonov regularization ${ }^{19}$ or truncated SVD ${ }^{20}$ are often employed to stabilize the computations. In recent years, several regularization formulations have also been explored in the context of the TLS problem. We distinguish between methods based on penalties/constraints, and methods based on truncation.

The basic idea of regularized total least squares $(R T L S)$ is forcing an upper bound on a weighted 2-norm of the solution vector $x$ (although other types of constraints can be envisaged). Several formulations have been considered. A first formulation is the quadratically constrained RTLS problem stated in Refs $21-24$ as

$$
\begin{aligned}
& \min _{x, \widehat{A}, \widehat{b}}\left\|\left[\begin{array}{ll}
A & b
\end{array}\right]-\left[\begin{array}{ll}
\widehat{A} & \widehat{b}
\end{array}\right]\right\|_{F}^{2} \\
& \quad \text { subject to } \widehat{A} x=\widehat{b},\|L x\|_{2}^{2} \leq \delta^{2},
\end{aligned}
$$

or, equivalently,

$$
\min _{x} \frac{\|A x-b\|_{2}^{2}}{1+\|x\|_{2}^{2}} \quad \text { subject to }\|L x\|_{2}^{2} \leq \delta^{2},
$$

where $L$ is a $p$ by $n$ matrix, usually the identity matrix or a discrete difference operator, and $\delta$ is a given scalar value.

A second formulation adds a Tikhonov-like quadratic penalty term $\|L x\|_{2}^{2}$ to the TLS objective function ${ }^{25}$ :

$$
\min _{x} \frac{\|A x-b\|_{2}^{2}}{1+\|x\|_{2}^{2}}+\lambda\|L x\|_{2}^{2} .
$$

For $\delta^{2}$ small enough (i.e., $\delta^{2}<\left\|L x^{\mathrm{TLS}}\right\|_{2}^{2}$ where $\hat{x}^{\mathrm{TLS}}$ is the TLS solution), there exists a value of the parameter $\lambda>0$ such that the solution of Eq. (10) coincides with the solution of Eq. (11). A sufficient condition for attainability of the minima in Eq. (10) or (11) is: $\sigma_{\min }\left(\left[\begin{array}{ll}A N & b\end{array}\right]\right)<\sigma_{\min }(A N)$, where the columns of $N$ form a basis for the nullspace of $L^{\top} L .^{22,24,25}$

As opposed to classical regularization methods in the context of ordinary least squares, these formulations do not have closed-form solutions. Although local optimization methods are used in practice, the analysis in Refs 24,25 suggests that both formulations can be recast in a global optimization framework, namely into scalar minimization problems, where each function evaluation requires the solution of a quadratically constrained linear least squares problem. ${ }^{26}$

The constrained formulation (10) has been solved via a sequence of quadratic eigenvalue problems by Ref 22. Combining this approach with the nonlinear Arnoldi method and reusing information from all previous quadratic eigenvalue problems, a more efficient method for large RTLS problems has been proposed in Ref 27. Further, Renaut and Guo ${ }^{23}$ suggested an iterative method based on a sequence of linear eigenvalue problems, which has also been accelerated by solving the linear eigenproblems by the nonlinear Arnoldi method and by a modified root finding method based on rational interpolation. ${ }^{28}$

For the quadratic penalty formulation (11), a complete analysis has been presented in Ref 25 . A simple reformulation into a scalar minimization makes the problem more tractable:

$$
\begin{aligned}
\min _{\alpha} \mathcal{G}(\alpha), \text { where } \mathcal{G}(\alpha) \\
\quad:=\min _{\|x\|_{2}^{2}=\alpha-1}\left\{\frac{\|A x-b\|_{2}^{2}}{\alpha}+\lambda\|L x\|_{2}^{2}\right\} .
\end{aligned}
$$

In Ref 29 another related formulation called dual RTLS is proposed. It minimizes the norm $\|L x\|_{2}^{2}$ subject to compatibility of the corrected system, as well as to upper bounds on $\|A-\widehat{A}\|_{\mathrm{F}}$ and $\|b-\widehat{b}\|_{2}$.

Truncation methods are another class of methods for regularizing linear ill-posed problems in the presence of measurement errors. In essence, they aim at limiting the contribution of noise or rounding errors by cutting off a certain number of terms in an SVD expansion. The truncated total least squares (TTLS) solution with truncation level $k$ is the minimum 2-norm solution of $A_{k} x=b_{k}$, where $\left[\begin{array}{ll}A_{k} & b_{k}\end{array}\right]$ is the best rank- $k$ approximation of $\left[\begin{array}{ll}A & b\end{array}\right]$. More precisely, if $U \Sigma V^{\top}$ is the SVD of [A $\left.\begin{array}{ll}A & b\end{array}\right]$,

$$
x_{\text {TTLS }, k}=-V_{12}^{k}\left(V_{22}^{k}\right)^{\dagger}=-V_{12}^{k}\left(V_{22}^{k}\right)^{\top} /\left\|V_{22}^{k}\right\|^{2},
$$

where we partition $V$ as (with $l=n-k+1$ ):

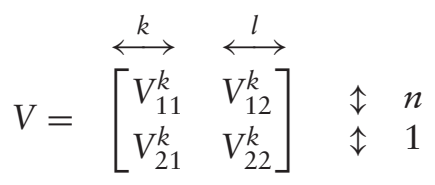

The regularizing properties of truncated total least squares and a filter factor expansion of the TTLS solution have been described in Ref 30. Sima and Van Huffel ${ }^{31}$ showed that the filter factors associated with the TTLS solution provide more information for 
choosing the truncation level compared with truncated SVD, where the filter factors are simply zeros and ones.

\section{APPLICATIONS AND CURRENT TRENDS}

Core problem: The concept of core problem in linear algebraic systems has been developed by Paige and Strakoš ${ }^{32}$. The idea is to find orthogonal $P$ and $Q$ such that

$$
P^{\top}\left[\begin{array}{ll}
b & A Q
\end{array}\right]=\left[\begin{array}{c||c|c}
b_{1} & A_{11} & 0 \\
\hline 0 & 0 & A_{22}
\end{array}\right] .
$$

The block $A_{11}$ is of full column rank, has simple singular values and $b_{1}$ has nonzero projections onto the left singular vectors of $A_{11}$. These properties guarantee that the subproblem $A_{11} x_{1} \approx b_{1}$ has minimal dimensions and contains all necessary and sufficient information for solving the original problem $A x \approx b$. All irrelevant and redundant information is contained in $A_{22}$.

Low-rank approximation: TLS problems aim at approximate solutions of overdetermined linear systems of equations $A X \approx B$. Typical application of TLS methods, however, are problems for data approximation by linear models. Such problems are mathematically equivalent to low-rank approximation, which in turn is not equivalent to the $A X \approx B$ problem. ${ }^{4}$ This suggests that from a data modeling point of view, a low-rank approximation is a better framework than the solution of an overdetermined linear system of equations. This viewpoint of the TLS data modeling approach is presented in Ref 3 .

Application of STLS in system identification and model reduction is described in Refs 10,33,34. Further applications of STLS include the shape from moments problem, ${ }^{35}$ approximate factorization and greatest common divisor computation in computer algebra, ${ }^{36}$ and image deblurring. ${ }^{37,38}$ The WTLS problem has applications in chemometrics ${ }^{14,15}$ and machine learning. ${ }^{39}$

Applications of RTLS: RTLS formulations, including weighted and structured generalizations, have been used in various ill-posed problems. A notorious inverse problem-blind deconvolution of oneor two-dimensional data-has received special attention. Restoring one-dimensional signals from noisy measurements of both the point-spread function and the observed data has been addressed by Refs 40,41 as a regularized structured TLS problem. A twodimensional generalization has been used for image restoration in Ref 42 . Interesting structured regularized problem formulations and efficient algorithms for image deblurring are analyzed in Refs 37,38,43-48. RTLS has also been used in image reconstruction of electrical capacitance tomography. ${ }^{49}$

Applications of TTLS: TTLS has successfully been applied to biomedical inverse problems such as the reconstruction of epicardial potentials from body surface potentials ${ }^{50}$ and imaging by ultrasound inverse scattering. ${ }^{51}$ TTLS is also used as an alternative to ridge regression in the estimation step of the regularized EM algorithm for the analysis of incomplete climate data. ${ }^{52}$

\section{ACKNOWLEDGEMENTS}

Diana Sima is postdoctoral fellow of FWO (Fund for Scientific Research-Flanders). Research supported by Research Council KUL: GOA MaNet, CoE EF/05/006 Optimization in Engineering (OPTEC), Belgian Federal Science Policy Office IUAP P6/04 (DYSCO), and PinView (Personal Information Navigator adapting through VIEWing), an EU FP7 funded Collaborative Project 216529.

\section{REFERENCES}

1. Van Huffel S, Zha H. The total least squares problem. In: Rao CR, ed. Handbook of Statistics: Computational Statistics, volume 9. Amsterdam: Elsevier Science; 1993, 377-408.

2. Van Huffel S. Total least squares and errors-in-variables modeling: bridging the gap between statistics, computational mathematics and engineering. In: Antoch J, ed.
COMPSTAT Proceedings in Computational Statistics. Heidelberg: Physika-Verlag, 2004, 539-555.

3. Markovsky I, Van Huffel S. Overview of total least squares methods. Signal Process 2007, 87:2283-2302.

4. Markovsky I. Structured low-rank approximation and its applications. Automatica 2007, 44:891-909. 
5. Van Huffel S, ed. Recent Advances in Total Least Squares Techniques and Errors-in-Variables Modeling. Philadelphia, PA: SIAM; 1997.

6. Van Huffel S, Lemmerling P, eds. Total Least Squares and Errors-in-Variables Modeling: Analysis, Algorithms and Applications. Dordrecht: Kluwer Academic Publishers, 2002.

7. Van Huffel S, Cheng C-L, Mastronardi N, Paige C, Kukush A. Editorial: total least squares and errorsin-variables modeling. Comput Stat Data Anal 2007, 52:1076-1079.

8. Van Huffel S, Markovsky I, Vaccaro RJ, Söderström T. Guest editorial: total least squares and errorsin-variables modeling. Signal Process 2007, 87 2281-2282.

9. Van Huffel S, Vandewalle J. The Total Least Squares Problem: Computational Aspects and Analysis. Philadelphia, PA: SIAM; 1991.

10. Markovsky I, Willems JC, Van Huffel S, De Moor B. Exact and Approximate Modeling of Linear Systems: A Behavioral Approach. Philadelphia: SIAM, 2006.

11. De Moor B. Structured total least squares and $L_{2}$ approximation problems. Linear Algebra Appl 1993, 188(189):163-207.

12. Manton J, Mahony R, Hua Y. The geometry of weighted low-rank approximations. IEEE Trans Signal Process 2003, 51:500-514.

13. Markovsky I, Van Huffel S. Left vs right representations for solving weighted low rank approximation problems. Linear Algebra Appl 2007, 422:540-552.

14. Wentzell P, Andrews D, Hamilton D, Faber K, Kowalski B. Maximum likelihood principal component analysis. J Chemometr 1997, 11:339-366.

15. Schuermans M, Markovsky I, Wentzell P, Van Huffel S. On the equivalence between total least squares and maximum likelihood PCA. Anal Chim Acta 2005, $544: 254-267$.

16. Golub G, Pereyra V. Separable nonlinear least squares: the variable projection method and its applications. Inverse Probl 2003, 19:1-26.

17. Abatzoglou TJ, Mendel IM, Harada GA. The constrained total least-squares technique and its applications to harmonic superresolution. IEEE Trans Signal Process 1991, 39:1070-1087.

18. Beck A, Ben-Tal A. A global solution for the structured total least squares problem with block circulant matrices. SIAM J Matrix Anal Appl 2006, 27:238-255.

19. Tikhonov AN, Arsenin V. Solutions of Ill-Posed Problems. Washington, D.C.: Winston \& Sons; 1977.

20. Hansen P-C. Truncated singular value decomposition solutions to discrete ill-posed problems with illdetermined numerical rank. SIAM J Sci Stat Comput 1990, 11:503-518.
21. Golub GH, Hansen PC, O’Leary DP. Tikhonov regularization and total least squares. SIAM J Matrix Anal Appl 1999, 21:185-194.

22. Sima DM, Van Huffel S, Golub GH. Regularized total least squares based on quadratic eigenvalue problem solvers. BIT Numer Math 2004, 44:793-812.

23. Renaut R, Guo H. Efficient algorithms for solution of regularized total least squares. SIAM J Matrix Anal Appl 2005, 26:457-476.

24. Beck A, Ben-Tal A, Teboulle M. Finding a global optimal solution for a quadratically constrained fractional quadratic problem with applications to the regularized total least squares. SIAM J Matrix Anal Appl 2006, 28:425-445.

25. Beck A, Ben-Tal A. On the solution of the Tikhonov regularization of the regularized total least squares problem. SIAM J Optim 2006, 17:98-118.

26. Gander W. Least squares with a quadratic constraint. Numer Math 1981, 36:291-307.

27. Lampe J, Voss H. On a quadratic eigenproblem occurring in regularized total least squares. Comput Stat Data Anal 2007, 52:1090-1102.

28. Lampe J, Voss H. A fast algorithm for solving regularized total least squares problems. Electron Trans Numer Anal 2008, 31:12-24.

29. Lu S, Pereverzev SV, Tautenhahn U. Dual regularized total least squares and multi-parameter regularization. Comput Meth Appl Math 2008, 8:253-262.

30. Fierro RD, Golub GH, Hansen PC, O'Leary DP. Regularization by truncated total least squares. SIAM J Sci Comput 1997, 18:1223-1241.

31. Sima DM, Van Huffel S. Level choice in truncated total least squares. Comput Stat Data Anal 2007, 52:1103-1118.

32. Paige CC, Strakoš Z. Core problems in linear algebraic systems. Comput Stat Data Anal 2005, 27:861-875.

33. Roorda B, Heij C. Global total least squares modeling of multivariate time series. IEEE Trans Automat Contr 1995, 40:50-63.

34. Markovsky I, Willems JC, Van Huffel S, Moor BD, Pintelon R. Application of structured total least squares for system identification and model reduction. IEEE Trans Automat Contr 2005, 50(10):1490-1500.

35. Schuermans M, Lemmerling P, Lathauwer LD, Van Huffel S. The use of total least squares data fitting in the shape from moments problem. Signal Process 2006, 86:1109-1115.

36. Botting B. Structured Total Least Squares for Approximate Polynomial Operations. Master's thesis, School of Computer Science, University of Waterloo, 2004.

37. Pruessner A, O'Leary DP. Blind deconvolution using a regularized structured total least norm approach. SIAM J Matrix Anal Appl 2003, 24:1018-1037. 
38. Mastronardi N, Lemmerling P, Van Huffel S. Fast regularized structured total least squares problems for solving the basic deconvolution problem. Numer Linear Algebra Appl 2005, 12:201-209.

39. Srebro N. Learning with Matrix Factorizations. PhD thesis, MIT, 2004.

40. Fan X. The Constrained Total Least Squares with Regularization and its Use in Ill-conditioned Signal Restoration. PhD thesis, Electrical, Electronic and Computer Engineering Department, Mississippi State University, 1992.

41. Younan NH, Fan X. Signal restoration via the regularized constrained total least squares. Signal Process 1998, 71:85-93.

42. Mesarović V, Galatsanos N, Katsaggelos A. Regularized constrained total least squares image restoration. IEEE Trans Image Process 1995, 4:1096-1108.

43. Kamm J, Nagy JG. Least squares and total least squares methods in image restoration. WNAA '96: Proceedings of the 1st International Workshop of Numerical Analysis and Its Applications. London: Springer-Verlag; 1997, 212-219.

44. Ng MK, Plemmons RJ, Pimentel F. A new approach to constrained total least squares image restoration. Linear Algebra Appl 2000, 316:237-258.

45. Chen W, Chen M, Zhou J. Adaptively regularized constrained total least-squares image restoration. IEEE Trans Image Process 2000, 9:588-596.
46. Mastronardi N, Lemmerling P, Kalsi A, O'Leary D, Van Huffel S. Implementation of the regularized structured total least squares algorithms for blind image deblurring. Linear Algebra Appl 2004, 391:203-221.

47. Kalsi A, O'Leary DP. Algorithms for structured total least squares problems with applications to blind image deblurring. J Res Natl Inst Stand Technol 2006, 111:113-119.

48. Fu H, Ng MK, Barlow JL. Structured total least squares for color image restoration. SIAM J Sci Comput 2006, 28:1100-1119.

49. Lei J, Liu S, Li Z, Schlaberg H, Sun M. An image reconstruction algorithm based on the regularized total least squares method for electrical capacitance tomography. Flow Meas Instrum 2008, 19:325-330.

50. Shou G, Xia L, Jiang M, Wei Q, Liu F, et al. Truncated total least squares: a new regularization method for the solution of ECG inverse problems. IEEE Trans Biomed Eng 2008, 55:1327-1335.

51. Liu C, Wang Y, Heng PA. A comparison of truncated total least squares with tikhonov regularization in imaging by ultrasound inverse scattering. Phys Med Biol 2003, 48:2437-2451.

52. Schneider T. Analysis of incomplete climate data: Estimation of mean values and covariance matrices and imputation of missing values. J Climate 2001, $14: 853-871$.

\section{FURTHER READING}

Beck A. The matrix-restricted total least-squares problem. Signal Process 2007, 87: 2303-2312.

Guo H. Renaut R. Parallel variable distribution for total least squares. Numer Linear Algebra Appl 2005, 12: 859-876.

Zhu W. Wang Y. Galatsanos N. P. Zhang J. Regularized total least squares approach for nonconvolutional linear inverse problems. IEEE Trans Image Process 1999, 8: 1657-1661. 